\title{
Evaluation of the InTray and Compact Dry culture systems for the diagnosis of urinary tract infections in patients presenting to primary health clinics in Harare, Zimbabwe
}

\author{
Ioana D. Olaru ${ }^{1,2}$ D $\cdot$ Wael Elamin ${ }^{3,4,5} \cdot$ Mutsawashe Chisenga $^{2} \cdot$ Nada Malou $^{6} \cdot$ Jeremie Piton $^{6} \cdot$ Shunmay Yeung $^{1,7}$. \\ Rashida A. Ferrand ${ }^{1,2} \cdot$ Heidi Hopkins ${ }^{1} \cdot$ Prosper Chonzi $^{8} \cdot$ Kudzai P. E. Masunda $^{8}$. Portia Manangazira ${ }^{9}$. \\ Cecilia Ferreyra ${ }^{6}$ Katharina Kranzer ${ }^{1,2,10}$
}

Received: 26 January 2021 / Accepted: 9 July 2021 / Published online: 22 July 2021

(c) The Author(s) 2021

\begin{abstract}
Antimicrobial resistance surveillance data is lacking from many resource-limited settings mainly due to limited laboratory testing. Novel culture systems may address some of the limitations of conventional culture media and expand the availability of microbiology services. The aims of this study were to evaluate the performance of InTray COLOREX Screen/ESBL and Compact Dry for the detection of uropathogens and of extended-spectrum beta-lactamase (ESBL)-producing organisms from urine samples. Urines samples were collected from patients presenting with symptoms of urinary tract infection to primary care clinics in Harare. Performance of the InTray COLOREX Screen, ESBL and Compact Dry chromogenic media were compared to the reference of culture using Brilliance UTI agar and conventional antimicrobial susceptibility testing. A total of 414 samples were included in the analysis. Of the included samples, 98 were positive on Brilliance UTI agar and 83 grew Enterobacterales. The sensitivities and specificities for Enterobacterales were 89.2\% (95\% CI 80.4-94.9) and 98.2\% (95\% CI 96.1-99.3) for InTray Screen and 95.2\% (95\% CI 88.1-98.7) and 99.7\% (95\% CI 98.3-100) for Compact Dry. Extendedspectrum beta-lactamases were present in 22 isolates from the Brilliance UTI agar. The sensitivity of the InTray COLOREX ESBL culture plates for the detection of ESBL-producing organisms was 95.5\% (95\% CI 77.2-99.9) and specificity was 99.5\% (95\% CI 98.2-99.9\%). Our findings show good performance of the novel culture systems for the detection of uropathogens and ESBL-producing organisms. Both systems have several advantages over conventional media and have the potential to expand and decentralize laboratory testing.
\end{abstract}

Keywords AMR $\cdot$ Antibiotic resistance $\cdot$ Enterobacterales $\cdot$ ESBL

\section{Introduction}

Antimicrobial resistance (AMR) represents a major threat to human health by impeding effective treatment of serious infections and leading to increased morbidity, mortality

Ioana D. Olaru

ioana-diana.olaru@1shtm.ac.uk

1 Clinical Research Department, London School of Hygiene and Tropical Medicine, Keppel Street, Bloomsbury, London WC1E 7HT, UK

2 Biomedical Research and Training Institute, Harare, Zimbabwe

3 G42 Healthcare, Abu Dhabi, United Arab Emirates

4 Queen Mary University London, London, UK and healthcare costs [1]. Acknowledging the implications of rising AMR, in 2015, the World Health Assembly adopted the Global Action Plan on AMR which outlined specific actions to address the increase in AMR including strengthening AMR surveillance and global data sharing

5 Elrazi University, Khartoum, Sudan

6 FIND (Foundation for Innovative New Diagnostics), Geneva, Switzerland

7 Department of Paediatric Infectious Disease, St. Mary's Imperial College Hospital, London, UK

8 City of Harare, Health Department, Harare, Zimbabwe

9 Ministry of Health and Child Care, Harare, Zimbabwe

10 Division of Infectious and Tropical Medicine, Medical Centre of the University of Munich, Munich, Germany 
[2]. In response to the Global Action Plan, the World Health Organization launched the Global AMR Surveillance System (GLASS).

The 2020 GLASS report emphasizes the persisting geographic gaps in AMR surveillance with health facilities and laboratories from Europe and the Americas contributing the majority of data. Data from low- and middleincome countries (LMIC) in Africa and Southeast Asia are scarce due to limited laboratory capacity and availability of microbiological diagnosis [3]. The main challenges restricting bacterial identification and detection of resistance are insufficient laboratory scientists and technicians, stock-outs of laboratory consumables, short shelf life of some key reagents, difficulties in supply chains management and prohibitive costs [4, 5]. In most LMICs, both availability and cost of diagnostic tests limit access for a large majority of patients who have most infectious conditions treated empirically and with the use of case definitions rather than laboratory confirmation. Blood cultures for example are usually restricted to tertiary referral hospitals and are not available at lower tier hospitals (i.e. district hospitals) and peripheral healthcare facilities [6].

Even at the levels where these tests are available, the turnaround time is such that antimicrobials are prescribed and consumed before the results are available to influence the choice. Limited availability of diagnostics results in a lack of AMR surveillance data and non-adaptation of empirical treatment to local pathogens and resistance profiles. Available data are usually biased towards more complex cases treated at referral centres or in private healthcare settings, and are not representative of the overall burden of resistance, challenging the development of locally adapted treatment recommendations.

Two novel ready-to-use culture systems, InTray and Compact Dry, may facilitate processing of urine samples in low-resource settings particularly in regional and district healthcare facilities where fully equipped microbiology laboratories may not be available. These systems have particular advantages that may allow for their use in lower tier facilities, thus expanding access to microbiology services. Urinary tract infections (UTIs) are common Gram-negative infections in outpatient settings. Organisms causing UTIs may provide valuable information on community-level Gram-negative resistance. Urine samples are non-invasive and considered priority specimens for AMR surveillance by the WHO-GLASS [3].

The aims of this study were to evaluate the performance of InTray COLOREX Screen/ESBL and Compact Dry for the detection of uropathogens and of extended-spectrum beta-lactamase (ESBL)-producing organisms in urine samples from patients presenting with UTI symptoms to primary care clinics in Harare.

\section{Methods}

Study participants were recruited into the antimicrobial resistance in Gram-negative bacteria from Urinary Specimens (ARGUS) study which is an observational crosssectional study enrolling adult patients who present with suspected UTIs to one of nine participating primary health clinics in Harare. The procedures and eligibility for the ARGUS study have been described in detail elsewhere [7]. Briefly, after obtaining informed consent, demographic and medical history was collected using a questionnaire and entered directly in an electronic form using the Open Data Kit (ODK, www.opendatakit.org). A midstream urine sample was collected from each of the study participants. If the transportation time to the laboratory was anticipated to exceed $4 \mathrm{~h}$, the urine sample was placed in a thermally insulated bag containing ice packs.

\section{Culture media}

This study used two novel culturing techniques-(1) InTrays COLOREX Screen and InTray COLOREX ESBL (Biomed Diagnostic, White City, OR, USA) and (2) Compact Dry EC (Nissui Pharmaceutical Co. Ltd., Tokyo, Japan) - and compared them to culture on Brilliance UTI agar (Oxoid, UK) which is considered the reference standard [8].

InTrays are ready-to-use commercially available smallsized agar plates (5-cm diameter for the agar) with chromogenic substrates to differentiate between multiple bacterial species based on colony colour. InTray COLOREX ESBL plates contain, in addition, antimicrobial compounds for selective identification of extended-spectrum beta-lactamase (ESBL)-producing organisms. InTrays have to be stored at $2-8{ }^{\circ} \mathrm{C}$ and have a shelf life of 6 (InTray COLOREX ESBL) to 12 months (InTray COLOREX Screen) [9].

Compact Dry EC is a dehydrated ready-to-use chromogenic medium designed for quantifying Escherichia coli and coliforms from food products. The media is hydrated by sample inoculation and capillary action allows for diffusion of the sample across the plate. The media contains two chromogenic enzyme substrates, Magenta-Gal and X-Gluc, which enable the differentiation between $E$. coli (blue) and other coliforms (pink). The plastic casing has a grid with large and small squares for ease of colony counting. For optimal colony counting, the manufacturer recommends diluting samples to concentrations of 100 colony forming units $(\mathrm{CFU}) / \mathrm{mL}$. The media is heat, light and moisture-sensitive and is supplied in small opaque pouches containing desiccant. The plates are stored at room temperature up to $30{ }^{\circ} \mathrm{C}$ and have a shelf life of 18 months post-manufacture [10]. 
Colony appearance and characteristics of the different media are described in Table 1 and additional Figures S1 and $\mathrm{S} 2$.

\section{Sample processing}

Following collection, the urine samples were inoculated by trained research assistants at the point of care onto the InTray COLOREX Screen and ESBL culture plates using a cotton swab (one tray per sample). For the Compact Dry and Brilliance UTI agar, urine samples were processed at the Biomedical Research and Training Institute research laboratory. The inoculated culture plates were then transported to the laboratory where they were incubated at $37^{\circ} \mathrm{C}$ for $24 \mathrm{~h}$. The InTrays were read after $24 \mathrm{~h}$ by a trained microbiologist. Information on the number of $\mathrm{CFU}$, morphology, colour and growth was recorded using a standardized form. Because InTrays require refrigeration, uninoculated plates had to be transported at the end of each working day to the laboratory and dispatched again to the clinics on the following day.

For Compact Dry, it was assumed that positive urine cultures would have a bacterial concentration of at least $10^{3} \mathrm{CFU} / \mathrm{mL}$ [11]. To perform colony counts, serial dilutions of 1:10 were performed on the day of sample collection. The urine samples were diluted up to $10^{6}$ in sterile phosphate-buffered saline and $1 \mathrm{~mL}$ each of the $10^{3}$ and $10^{6}$ dilutions was inoculated on the Compact Dry and incubated for $24 \mathrm{~h}$ at $37^{\circ} \mathrm{C}$. The optimal dilutions were established during a pilot phase and previous use of the culture system in our laboratory (unpublished). The plates were read by a trained microbiologist and the number of CFU and colony appearance was recorded. If colonies could not be counted, growth was categorized semi-quantitatively into semi-confluent (some individual colonies still visible) and confluent growth (individual colonies not visible with a change in substrate colour).

Brilliance UTI agar was inoculated at the laboratory using $1-\mu \mathrm{L}$ sterile loops and incubated at $37{ }^{\circ} \mathrm{C}$ for $24 \mathrm{~h}$. Growth was reported semi-quantitatively into three categories: $10^{3}-10^{4} \mathrm{CFU} / \mathrm{mL}, 10^{4}-10^{5} \mathrm{CFU} / \mathrm{mL}$ and $>10^{5} \mathrm{CFU} /$ $\mathrm{mL}$. All cultures showing growth of $>10^{3} \mathrm{CFU} / \mathrm{mL}$ with the predominance of uropathogens were considered positive.

Antimicrobial susceptibility testing (AST) was done by disc diffusion and interpreted according to the EUCAST standards [12]. ESBL testing of Enterobacterales was performed according to the EUCAST recommendations [13]. Briefly, isolates were screened for the presence of ESBLs using cefpodoxime. Isolates positive on the screening test underwent confirmation by synergy testing with amoxicillin/clavulanic acid and ceftazidime. Quality control for bacterial identification and AST was performed using ATCC reference isolates.

Table 1 Colony appearance and product characteristics for the three culture systems used in the study [8-10]

\begin{tabular}{|c|c|c|c|}
\hline & Brilliance UTI agar & InTray COLOREX & Compact Dry EC \\
\hline E. coli & Pink & Pink & Blue \\
\hline KESC* & Dark blue & Blue/turquoise & Red-violet (pink) \\
\hline Proteus group & Brown halo & Brown halo & Yellow-brown \\
\hline Pseudomonas spp. & Brown/green & Cream, translucent & - \\
\hline Enterococcus spp. & Turquoise & Blue/turquoise & Inhibited \\
\hline S. aureus & White/cream & Golden & Inhibited \\
\hline S. saprophyticus & Pink, small & Pink, small & Inhibited \\
\hline Other streptococci/staphylococci & Non-pigmented/white & White & Inhibited \\
\hline \multicolumn{4}{|l|}{ Product and use characteristics } \\
\hline Storage temperature & $\begin{array}{l}\text { Dehydrated: RT } \\
\text { Prepared: } 2-8{ }^{\circ} \mathrm{C}\end{array}$ & $2-8{ }^{\circ} \mathrm{C}$ & $\mathrm{RT}\left(<30^{\circ} \mathrm{C}\right)$ \\
\hline Shelf life & $\begin{array}{l}\text { Dehydrated: } \sim 2 \text { years } \\
\text { Prepared: } 2 \text { weeks }\end{array}$ & $6-12$ months & 18 months \\
\hline Size/format (mm) & $\begin{array}{l}\text { Dehydrated: container } \\
\text { Prepared: } 85 \times 85 \times 14\end{array}$ & $\begin{array}{l}103 \times 75 \times 8 \\
\text { Agar: } 5 \mathrm{~cm}\end{array}$ & Culture media: $5.5 \mathrm{~cm}$ \\
\hline Number of samples & Multiple & Single & Single \\
\hline Preparation required & Yes (dehydrated) & No & No \\
\hline Number of samples inoculated & $1-6 /$ plate & 1 & 1 \\
\hline Sample and volume inoculated & Neat urine, $1 \mu \mathrm{L}$ & Neat urine, using a swab & Diluted urine $10^{3}$ and $10^{6} ; 1 \mathrm{~mL}$ \\
\hline Location of sample inoculation & Laboratory & Point of care (clinic) & Laboratory \\
\hline
\end{tabular}

KESC Klebsiella, Enterobacter, Serratia, Citrobacter; RT room temperature. Dehydrated media, supplied in powder form requiring preparation; prepared, pre-poured plates as supplied by the manufacturer 


\section{Experience in using the tests}

Research assistants and laboratory staff involved in sample processing were asked about the perceived advantages and shortcomings of the test systems. The number of times the InTray plates were removed from the fridge and dispatched to the clinics was recorded to determine if multiple exposures to high temperatures affect the test performance.

\section{Statistical analysis}

Descriptive analyses were performed in STATA v.15 (StataCorp, TX, USA). To evaluate test performance, sensitivity and specificity with $95 \%$ confidence intervals $(95 \% \mathrm{CI}$ ) were calculated. The correlation between CFUs on Compact Dry and growth on Brilliance UTI agar categorized semi-quantitatively was evaluated using Spearman's correlation test.

The study was granted ethics approval by the London School of Hygiene and Tropical Medicine (ref. 16,424) and by the Medical Research Council of Zimbabwe (MRCZ/A/2406).

\section{Results}

A total of 431 urine samples were tested using the three culture systems. Of those, 17 (3.9\%) were contaminated on Brilliance UTI agar and were excluded leaving 414 samples for the final analysis. The median age of study participants was 36 years (IQR 26-46), 263 (63.5\%) were female and 169 (42.7\%) were HIV positive. Participant characteristics are shown in additional Table S1.

\section{InTray COLOREX Screen for uropathogen detection}

Using the Brilliance UTI agar, 98 urine cultures were positive. Uropathogens identified were 72 E. coli $(73.5 \%), 11$ other Enterobacterales (11.2\%), 13 Enterococcus spp. (13.3\%) and two Staphylococcus aureus (2.0\%). Of the 72 cultures growing $E$. coli on the Brilliance UTI agar, 64 showed growth of pink colonies (E. coli) on the InTray COLOREX Screen culture plates while eight were positive on Brilliance only and six on InTrays only. For other coliforms, ten cultures were positive on both Brilliance and InTrays and an additional sample was positive on the Brilliance UTI agar only (Table 2).

Of the 13 samples that were positive on the Brilliance UTI agar for enterococci, nine were also positive for enterococci on the InTray COLOREX Screen cultures. The four discordant samples that were positive for enterococci on the Brilliance UTI agar had growth of enterococci on the InTrays but enterococci were not the dominant organism. Conversely, 15 cultures had predominant growth of enterococci on the InTray but were negative on the Brilliance UTI agar. The two cultures with growth of $S$. aureus were positive on both culture media.

Using the Brilliance UTI agar as the reference standard and a threshold for positivity of $10^{3} \mathrm{CFU} / \mathrm{mL}$, the sensitivity of InTray COLOREX Screen for detecting Enterobacterales was $89.2 \%$ (74/83; 95\% CI 80.4-94.9) and the specificity was $98.2 \%$ (325/331; 95\% CI 96.1-99.3). Positive and negative predictive values were $92.5 \%$ (74/80; 95\% CI 84.4-97.2) and $97.3 \%$ (325/334; 95\% CI 94.9-98.8), respectively. Test performance according to different thresholds for culture positivity is shown in Table 3 .

\section{Compact Dry EC for uropathogen detection}

Of the 83 samples which had growth of Enterobacterales on Brilliance UTI agar, 79 had growth on the Compact Dry EC of Enterobacterales at the $1: 10^{3}$ dilution and 58 at the $1: 10^{6}$ dilution. Four samples which were positive for E. coli on Brilliance were negative on Compact Dry and one sample with Proteus spp. was positive on

Table 2 Comparison between culture results for InTray Screen and Brilliance UTI agar for Enterobacterales

\begin{tabular}{|c|c|c|c|c|c|c|}
\hline & & \multicolumn{4}{|c|}{ Brilliance UTI agar } & \multirow[t]{2}{*}{ Total } \\
\hline & & $\begin{array}{l}\text { Negative for } \\
\text { coliforms }{ }^{\#}\end{array}$ & $\begin{array}{l}10^{3}-10^{4} \mathrm{CFU} / \\
\mathrm{mL}\end{array}$ & $\begin{array}{l}10^{4}-10^{5} \mathrm{CFU} / \\
\mathrm{mL}\end{array}$ & $>10^{5} \mathrm{CFU} / \mathrm{mL}$ & \\
\hline \multirow[t]{6}{*}{ InTray COLOREX Screen } & Negative (or $1-4$ colonies) ${ }^{*}$ & 325 & 4 & 3 & 2 & 334 \\
\hline & Positive & & & & & \\
\hline & 5-49 colonies & 4 & 5 & 3 & 0 & 12 \\
\hline & 50-100 colonies & 0 & 1 & 0 & 1 & 2 \\
\hline & Confluent growth & 2 & 0 & 8 & 56 & 66 \\
\hline & Total & 331 & 10 & 14 & 59 & 414 \\
\hline
\end{tabular}

\footnotetext{
*Negative cultures, one colony on InTray ESBL but not on Screen $(n=2)$; mixed growth of organisms with colonies on InTray but $<5$ colonies $(n=3) ;{ }^{\#} 3 / 6$ discordant cultures with a positive InTray also had growth with low colony count on Compact Dry. Gram-positive organisms $($ Enterococcus spp., $n=13$ and $S$. aureus, $n=2$ ) were not included in the table as their growth is inhibited on the Compact Dry media
} 
Table 3 Performance for Compact Dry EC and InTray Screen in the detection of Enterobacterales compared with Brilliance UTI agar and using different thresholds for culture positivity on Brilliance agar

\begin{tabular}{clcccc}
\hline & $\begin{array}{l}\text { Cut-off on Bril- } \\
\text { liance agar }\end{array}$ & Sensitivity (95\% CI) & Specificity (95\% CI) & PPV (95\% CI) & NPV (95\% CI) \\
\hline Compact Dry at & $10^{3}$ & $95.2(88.1-98.7)$ & $99.7(98.3-100)$ & $98.8(93.2-99.9)$ & $98.8(97.0-99.7)$ \\
$1: 10^{3}$ dilution & $10^{4}$ & $98.6(92.6-100)$ & $97.7(95.4-99.0)$ & $90.0(81.2-95.6)$ & $99.7(98.3-99.9)$ \\
& $10^{5}$ & $100(93.9-100)$ & $94.1(91.1-96.3)$ & $73.8(62.7-83.0)$ & $100(98.9-100)$ \\
InTray COLO- & $10^{3}$ & $89.2(80.4-94.9)$ & $98.2(96.1-99.3)$ & $92.5(84.4-97.2)$ & $97.3(94.9-98.8)$ \\
REX Screen & $10^{4}$ & $93.2(84.7-97.7)$ & $96.5(93.9-98.2)$ & $85.0(75.2-92.0)$ & $98.5(96.5-99.5)$ \\
& $10^{5}$ & $96.6(88.3-100)$ & $93.5(90.4-95.8)$ & $71.3(60.0-80.8)$ & $99.4(97.9-99.9)$ \\
\hline
\end{tabular}

$N P V$ negative predictive value; $P P V$ positive predictive value

Compact Dry only (Table 4). The sensitivity and specificity for uropathogen detection were $95.2 \%$ (79/83; $95 \%$ CI 88.1-98.7) and 99.7\% (330/331; 95\% CI 98.3-100), respectively, using a threshold for urine culture positivity of $10^{3} \mathrm{CFU} / \mathrm{mL}$ on the Brilliance UTI agar (Table 3). Positive and negative predictive values were $98.8 \%$ (79/80; 95\% CI 93.2-99.9) and 98.8\% (330/334; 95\% CI 97.0-99.7). Using the $1: 10^{3}$ dilution, $55 / 80$ (69\%) of positive samples on Compact Dry had semi-confluent or confluent growth and therefore colony counts could not be performed. While the $1: 10^{3}$ dilution had a better sensitivity, the higher dilution allowed for colony counts. There was a strong correlation between CFUs determined using the Compact Dry and the semi-quantitative assessment on Brilliance UTI agar (Spearman's rho 0.924, $p<0.001$; Fig. 1).

\section{InTray COLOREX ESBL for the diagnosis of cephalosporin-resistant organisms}

InTray COLOREX ESBL culture results were available for 413 samples. Synergy testing for the presence of ESBLs was positive in 22/24 isolates tested (E. coli, $n=20$; other coliforms, $n=2$ ). Of the $22 \mathrm{ESBL}$-positive organisms using conventional methods, 21 were positive on InTray COLOREX ESBL. Two samples were positive on InTray COLOREX ESBL only: negative on Brilliance UTI agar $(n=1)$; negative screening test for ESBL using cefpodoxime $(n=1)$. One sample which was ESBL positive using conventional methods and negative on InTray had growth of a single colony on InTray. The sensitivity of the InTray COLOREX ESBL culture plates for the detection of ESBL-producing organisms was $95.5 \%(21 / 22$; 95\% CI 77.2-99.9) and specificity was $99.5 \%$ (400/402; 95\% CI 98.2-99.9\%).

Table 4 Comparison between culture results for Compact Dry and Brilliance UTI agar for Enterobacterales

\begin{tabular}{|c|c|c|c|c|c|c|}
\hline & & \multicolumn{4}{|c|}{ Brilliance UTI agar } & \multirow[t]{2}{*}{ Total } \\
\hline & & $\begin{array}{l}\text { Negative for } \\
\text { coliforms }\end{array}$ & $\begin{array}{l}10^{3}- \\
10^{4} \mathrm{CFU} / \\
\mathrm{mL}\end{array}$ & $\begin{array}{l}10^{4}- \\
10^{5} \mathrm{CFU} / \\
\mathrm{mL}\end{array}$ & $\begin{array}{l}>10^{5} \mathrm{CFU} / \\
\mathrm{mL}\end{array}$ & \\
\hline \multirow[t]{5}{*}{ Compact Dry EC at $1: 10^{3}$ dilution } & Negative (or $1-4$ colonies) & 330 & 3 & 1 & 0 & 334 \\
\hline & 5-49 colonies & 0 & 6 & 3 & 2 & 11 \\
\hline & $50-250$ colonies & 0 & 1 & 4 & 2 & 7 \\
\hline & Semi-confluent growth or $>250$ colonies & 0 & 0 & 6 & 18 & 24 \\
\hline & Confluent growth & $1 *$ & 0 & 0 & 37 & 38 \\
\hline \multirow[t]{6}{*}{ Compact Dry EC at $1: 10^{6}$ dilution } & Negative & 331 & 8 & 11 & 6 & 356 \\
\hline & $1-49$ colonies $^{\#}$ & 0 & 2 & 3 & 32 & 37 \\
\hline & $50-250$ colonies & 0 & 0 & 0 & 18 & 18 \\
\hline & Semi-confluent growth or $>250$ colonies & 0 & 0 & 0 & 2 & 2 \\
\hline & Confluent growth & 0 & 0 & 0 & 1 & 1 \\
\hline & Total & 331 & 10 & 14 & 59 & 414 \\
\hline
\end{tabular}

Cultures which showed contamination on Brilliance UTI agar were excluded; *this culture had mixed growth and one of the organisms was Proteus which had confluent growth on the Compact Dry due to swarming; ${ }^{\#}$ for the 1:10 ${ }^{6}$ dilution, this category included growth of $1-49$ colonies 
Fig. 1 Comparison of semiquantitative bacterial growth on Brilliance UTI agar and colony counts using Compact Dry

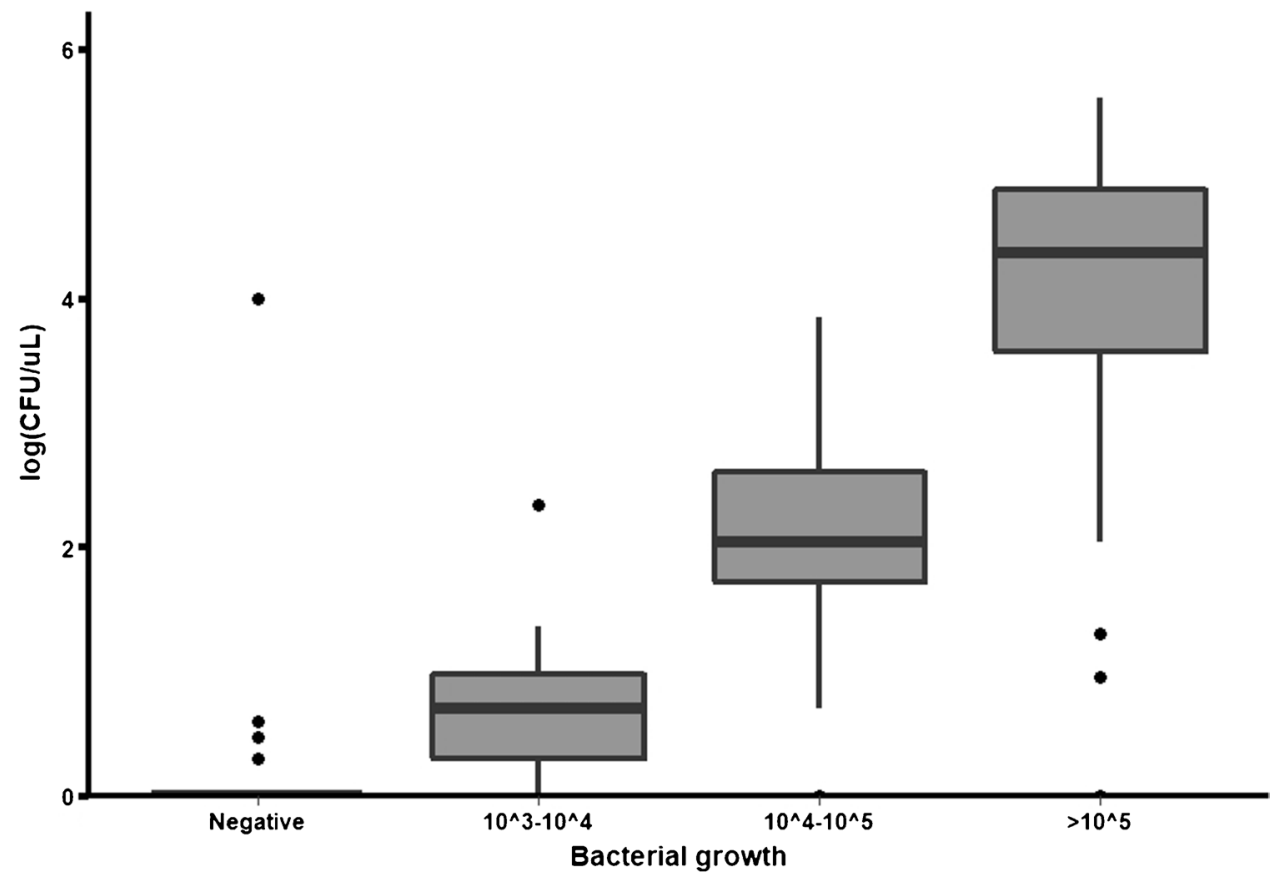

\section{Reported experience of staff using the tests}

Research assistants who inoculated the urine samples onto InTrays at point of care $(n=9)$ reported that overall the diagnostic device was easy to use, and they reported no challenges inoculating the agar in the field. The procedure was simple and was not time-consuming. The main challenge was the requirement for the InTrays to be refrigerated and thus InTrays not used on a particular day had to be transported daily from the laboratory to the clinics. In order to decrease the number of times the InTrays were transported between the laboratory and clinics, a limited number of InTrays was issued to each clinic every day. This meant that research assistants sometimes ran out of InTrays if the number of participants enrolled outnumbered the number of InTrays sent to the clinic. The median number of times InTrays were sent to the clinics was 2 (IQR 1-4). The number of times an InTray was transported to a clinic did not affect sensitivity of the culture system.

Laboratory staff $(n=2)$ reported on the processing, reading and interpretation of the test results. Both InTray and Compact Dry plates are relatively small in size minimizing incubator space requirements. For both systems, cultures were very easy to read and interpret. A major advantage compared to the UTI Brilliance agar was that InTrays and Compact Dry were ready to use and did not require media preparation and autoclaving. In LMICs, electricity supplies can be unreliable and generators cannot always provide a sufficient power supply for high-energy processes such as autoclaving. In the study setting, media preparation and autoclaving could not be done during power-cuts. However,
Compact Dry was more difficult to inoculate because they required sequential dilutions which were performed in the laboratory.

\section{Discussion}

Both novel culture systems performed well in detecting uropathogens compared to Brilliance UTI chromogenic media, the reference standard. The diagnostic systems were easy to use and study staff required minimal training for inoculation and reading. Furthermore, InTray COLOREX ESBL plates showed a high sensitivity and specificity for the detection of ESBL Enterobacterales making them an attractive tool for AMR surveillance in LMICs. Because the system selectively identifies ESBL-producing organisms from primary specimens, it can reduce time to results when used for diagnosis, which in turn may reduce time to appropriate therapy.

Conventional culture media have to be prepared frequently and require refrigeration which may be challenging in LMICs due to unreliable electricity supplies and equipment maintenance. Preparation of dehydrated media requires autoclaving and quality control of every prepared batch. InTray and Compact Dry are pre-prepared culture plates which are smaller than conventional plates and have an extended shelf life bypassing some of the shortcomings associated with conventional cultures. Both systems are based on chromogenic identification. Although chromogenic media for bacterial identification are not novel and have been in use for more than two decades [14], media 
prepared in-house from dehydrated powder usually expire within 2 weeks [8]. InTray COLOREX Screen and ESBL are stable for 6-12 months and Compact Dry EC for 18 months post-manufacture. Furthermore, Compact Dry media does not require refrigeration and can be stored at temperatures up to $30^{\circ}$ which is an added advantage when used in LMICs.

The Compact Dry system has been designed for determining bacterial contamination of food [15]. One previous study from Japan reported good sensitivity of the Compact Dry when used to investigate UTIs in humans, but the sample size was small $(n=25)$ [16]. Because the Compact Dry is a highly sensitive culture system and positive urine cultures usually have high bacterial loads, the urine samples were diluted prior to inoculation. For the purpose of this study, serial dilutions were performed. However, the procedures could be simplified by performing a single dilution at a higher factor (for example by diluting 1- $\mu \mathrm{L}$ sample into a $1 \mathrm{~mL}$ of sterile solution). A volume of $1 \mathrm{~mL}$ is required in order to rehydrate the dry culture media. Although of limited clinical significance, Compact Dry allows for colony counts which can be used to estimate bacterial load in samples. In this study, colony counts using the Compact Dry correlated well with the semi-quantitative results on Brilliance UTI agar.

Gram-positive pathogens such as Enterococcus spp. and S. saprophyticus account for $15 \%$ of uncomplicated UTIs [17]. While InTrays can detect these pathogens, Compact Dry ECs can only be used for the detection of Enterobacterales and do not support growth of Gram-positive organisms. Enterococci were detected more frequently using InTrays than on conventional media. This may suggest an under-diagnosis of enterococcal infections using conventional media although growth of enterococci from midstream urine samples may not necessarily reflect the presence of enterococci in the urinary bladder [18] and therefore results should be interpreted with caution considering the patient's medical history.

Cultures that were positive on InTray only may be explained by inoculation of a larger sample volume using swabs compared to $1-\mu \mathrm{L}$ inoculation on Brilliance UTI agar. Furthermore, immediate inoculation of the sample may have prevented loss of bacterial viability during transport. Falsenegative Compact Dry results were only observed for urine samples with lower bacterial load classified as $10^{3}-10^{4}$ CFUs on the Brilliance UTI agar. These urine samples may have shown growth on Compact Dry if the urine had been less diluted $\left(<10^{3}\right)$. One sample showed confluent growth for Proteus spp. on the Compact Dry but was negative on Brilliance UTI agar. This was due to the inhibition of swarming on the Brilliance agar but not on Compact Dry.

This is the first study evaluating the performance of the InTray and Compact Dry culture systems for the diagnosis of UTI. We acknowledge that the study is limited by its relatively small sample size and low proportion of positive urine cultures.

Our findings show good performance of the novel culture systems for the detection of uropathogens and ESBL-producing organisms. These systems may simplify laboratory workflow, reduce technician processing time and facilitate procurement and stock management. Both systems have potential to expand and decentralize laboratory testing. Use of the systems in sentinel clinical sites may enhance understanding of pathogens and AMR burden in LMICs. Further research is needed to demonstrate cost-effectiveness and feasibility of wider implementation of these systems for AMR surveillance, and potentially impact on patient outcomes in LMICs.

Supplementary Information The online version contains supplementary material available at https://doi.org/10.1007/s10096-021-04312-4.

Acknowledgements This work was supported by the Foundation for Innovative New Diagnostics (FIND) who provided the InTray COLOREX Screen and ESBL for evaluation.

Author contribution IDO and KK conceived the study idea and planned the study. WE, NM, JP and CF contributed to the study idea. $\mathrm{NM}, \mathrm{JP}, \mathrm{CF}, \mathrm{PC}$ and KPEM provided support with the resources needed for the study. SY, RAF and $\mathrm{HH}$ provided support with conducting the study. IDO and MC conducted the laboratory testing and interpretation of results. IDO and $\mathrm{KK}$ analysed and interpreted the data, and drafted the initial manuscript. WE, MC, NM, JP, SY, RAF, HH, PC, KPEM and $P M$ reviewed and provided feedback on the initial manuscript. All authors read and approved the final manuscript.

Funding IDO received funding though the Wellcome Trust Clinical $\mathrm{PhD}$ Programme awarded to the London School of Hygiene and Tropical Medicine (grant number 203905/Z/16/Z). The funders had no role in study design, data collection and interpretation, or the decision to submit the work for publication. The study was funded by UK aid from the UK government; the views expressed, however, do not necessarily reflect the UK government's official policies. FIND was supported by UK aid from the British people for the acquisition of the InTrays which were used in the study.

Data availability The datasets generated and/or analysed during the current study are available in the Data Compass (LSHTM) repository, https://datacompass.lshtm.ac.uk/1997/. All data generated or analysed during this study are included in this published article and its supplementary information files.

Code availability Not applicable.

\section{Declarations}

Ethics approval The study was performed in accordance with the ethical standards as laid down in the 1964 Declaration of Helsinki and its later amendments or comparable ethical standards. The study was granted ethics approval by the London School of Hygiene and Tropical Medicine (ref. 16424) and by the Medical Research Council of Zimbabwe (MRCZ/A/2406). 
Consent to participate All study participants provided written informed consent prior to study participation.

Consent for publication Not applicable.

Competing interests The authors declare no competing interests.

Open Access This article is licensed under a Creative Commons Attribution 4.0 International License, which permits use, sharing, adaptation, distribution and reproduction in any medium or format, as long as you give appropriate credit to the original author(s) and the source, provide a link to the Creative Commons licence, and indicate if changes were made. The images or other third party material in this article are included in the article's Creative Commons licence, unless indicated otherwise in a credit line to the material. If material is not included in the article's Creative Commons licence and your intended use is not permitted by statutory regulation or exceeds the permitted use, you will need to obtain permission directly from the copyright holder. To view a copy of this licence, visit http://creativecommons.org/licenses/by/4.0/.

\section{References}

1. Cosgrove SE (2006) The relationship between antimicrobial resistance and patient outcomes: mortality, length of hospital stay, and health care costs. Clin Infect Dis 42 Suppl 2:S82-89

2. World Health Organization (2015) Global action plan on antimicrobial resistance. WHO. http://www.wpro.who.int/entity/drug resistance/resources/global_action_plan_eng.pdf. Accessed 15 Jan 2021

3. Global antimicrobial resistance surveillance system (GLASS) report: early implementation (2020). Geneva: World Health Organization. Licence: CC BY-NC-SA 3.0 IGO. Available from: https://www.who.int/glass/resources/publications/early-imple mentation-report-2020/en/. Accessed 15 Jan 2021

4. Boeras DI, Peeling RW, Onyebujoh P, Yahaya AA, GumedeMoeletsi HN, Ndihokubwayo JB (2016) The WHO AFRO external quality assessment programme (EQAP): linking laboratory networks through EQA programmes. Afr J Lab Med 5(2):560

5. Usuf E, Mackenzie G, Sambou S, Atherly D, Suraratdecha C (2016) The economic burden of childhood pneumococcal diseases in The Gambia. Cost Eff Resour Alloc 14:4

6. Crump JA, Heyderman RS (2015) A perspective on invasive Salmonella disease in Africa. Clin Infect Dis 61 Suppl 4:S235-240

7. Olaru ID, Yeung S, Ferrand RA, Stabler R, Chonzi P, Mabey D et al (2020) Antimicrobial resistance in gram-negative bacteria from urinary specimens: a study of prevalence, risk factors and molecular mechanisms of resistance (ARGUS) in Zimbabwe - a study protocol. Wellcome Open Research 5:140
8. Brilliance UTI culture media (Oxoid). Product information. Available from http://www.oxoid.com/uk/blue/prod_detail/prod_detail. asp? $\mathrm{pr}=\mathrm{cm} 0949 \& \mathrm{c}=\mathrm{uk} \& \mathrm{lang}=\mathrm{en}$. Accessed 15 Jan 2021

9. InTray COLOREX Screen and ESBL product information. Available from https://biomeddiagnostics.com/prepared-cultu re-media/clinical-microbiology/catalog/hospital-acquired-infec tions. Accessed 15 Jan 2021

10. Compact Dry EC Product information sheet and instructions for use. Available from https://catalog.hardydiagnostics.com/cp_ prod/Content/hugo/CompactDryEC.pdf. Accessed 15 Jan 2021

11. Bonkat G, Pickard R, Bartoletti R et al EAU guidelines on urological infections. Arnhem, the Netherlands: European Association of Urology. Available from https://uroweb.org/wp-content/uploa ds/EAU-Guidelines-on-Urological-Infections-2018-large-text. pdf. Accessed 15 Jan 2021.

12. The European Committee on Antimicrobial Susceptibility Testing. Breakpoint tables for interpretation of MICs and zone diameters. Version 8.1, 2018. Available from https://www.eucast.org/filea $\mathrm{dmin} / \mathrm{src} / \mathrm{media} / \mathrm{PDFs} /$ EUCAST_files/Breakpoint_tables/v_8.1_ Breakpoint_Tables.pdf. Accessed 15 Jan 2021

13. EUCAST guidelines for detection of resistance mechanisms and specific resistances of clinical and/or epidemiological importance Version 2.01 July 2017. Available from https://eucast.org/resis tance_mechanisms/. Accessed 15 Jan 2021

14. Samra Z, Heifetz M, Talmor J, Bain E, Bahar J (1998) Evaluation of use of a new chromogenic agar in detection of urinary tract pathogens. J Clin Microbiol 36(4):990-994

15. Mizuochi S, Nelson M, Baylis C, Jewell K, Green B, Limbum R, Fernandez MC, Salfinger Y, Chen Y (2016) Matrix extension study: validation of the Compact Dry TC method for enumeration of total aerobic bacteria in selected foods. J AOAC Int 99(2):461-468

16. Horii T, Morita M, Kameno Y, Kanno T, Maekawa M (2002) Comparison of a new system (Compactdry SCD) with conventional methods for quantitative urine cultures. Lett Appl Microbiol 35(6):499-503

17. Flores-Mireles AL, Walker JN, Caparon M, Hultgren SJ (2015) Urinary tract infections: epidemiology, mechanisms of infection and treatment options. Nat Rev Microbiol 13(5):269-284

18. Hooton TM, Roberts PL, Cox ME, Stapleton AE (2013) Voided midstream urine culture and acute cystitis in premenopausal women. N Engl J Med 369(20):1883-1891

Publisher's note Springer Nature remains neutral with regard to jurisdictional claims in published maps and institutional affiliations. 